\title{
Effect of Scopolamine Butylbromide on Clozapine-induced Hypersalivation in Schizophrenic Patients: A Case Series
}

\author{
Ippei Takeuchi ${ }^{1}, 2$, Tatsuyo Suzuki ${ }^{1}$, Taro Kishi ${ }^{1}$, Daisuke Kanamori ${ }^{1}$, Manako Hanya ${ }^{2}$, Junji Uno ${ }^{1}$, Kiyoshi Fujita', \\ Hiroyuki Kamei \\ ${ }^{1}$ Department of Psychiatry, Okehazama Hospital, Toyoake, Aichi, ${ }^{2}$ Office of Clinical Pharmacy Practice and Health Care Management \\ Faculty of Pharmacy, Meijo University, Nagoya, Japan
}

\begin{abstract}
Clozapine has been demonstrated to be useful for treating refractory schizophrenia. However, hypersalivation occurs in 31.0$97.4 \%$ of the patients treated with clozapine. Accordingly, some patients who are disturbed by their hypersalivation refuse to continue with clozapine treatment. This study investigated the efficacy of the anticholinergic agent scopolamine butylbromide against clozapine-induced hypersalivation. Five schizophrenia patients were coadministered scopolamine butylbromide (30-60 mg/ day) for 4 weeks. At the baseline and after 4 weeks' treatment, we subjectively evaluated hypersalivation using a visual analog scale and objectively assessed it using the Drooling Severity Scale and Drooling Frequency Scale. As a result, improvements in the patients' Drooling Severity Scale and Drooling Frequency Scale scores, but no improvements in their visual analog scale scores, were observed after scopolamine butylbromide treatment. These results indicate that at least some schizophrenic patients with clozapine-induced hypersalivation would benefit from scopolamine butylbromide treatment. We conclude that clozapine-induced hypersalivation is one factor of stress to patients. Subjective hypersalivation was not improved, but objective hypersalivation was, by scopolamine butylbromide treatment. However, scopolamine butylbromide and clozapine possess anticholinergic effects so clinicians should closely monitor patients who take scopolamine butylbromide.
\end{abstract}

KEY WORDS: Clozapine; Schizophrenia; Hypersalivation; Sialorrhea; Scopolamine butylbromide.

\section{INTRODUCTION}

Clozapine (CLZ) has a superior ability in the treatment of refractory schizophrenia, but it has agranulocytosis as an emergent side-effect. Therefore, the side effects of CLZ, in particular agranulocytosis, continue to be a focus of concern. Other adverse effects, such as hypersalivation, sedation, and constipation, are usually less severe, but can have significant impacts on tolerability, medication adherence, and quality of life (QOL). ${ }^{1)}$ Hypersalivation occurs in $31.0-97.4 \%$ of patients treated with CLZ.,3) It usually develops early in the course of treatment, but physicians often take longer to recognize hypersalivation than they do to detect agranulocytosis or other side effects. ${ }^{4)}$ For this reason, some patients find it difficult to continue with CLZ treatment. Although the mechanisms responsible for CLZ-

\footnotetext{
Received: October 8, 2014 / Revised: November 15, 2014

Accepted: November 19, 2014

Address for correspondence: Ippei Takeuchi, M. Pharm Department of Psychiatry, Okehazama Hospital, Minamiyakata 3-879, Sakae-cho, Toyoake, Aichi 470-1168, Japan

Tel: +81-562-97-1361, Fax: +81-562-97-8004

E-mail: 134331503@ccalumni.meijo-u.ac.jp
}

induced hypersalivation remain unknown, it has been suggested that CLZ's partially agonistic stimulation of the M1 receptor $^{5)}$ and agonistic stimulation of the M4 receptor in the salivary glands lead to increased saliva secretion. ${ }^{6}$ )

Previous reports have indicated that the following anticholinergic agents are effective against hypersalivation; scopolamine, ${ }^{7)}$ ipratropium bromide, ${ }^{8)}$ pirenzepine, ${ }^{9)}$ trihexyphenidyl, ${ }^{10,11)}$ and oxybutynin. ${ }^{12)}$ Anticholinergic agents block the effects of muscarinic antagonists on smooth muscle. Common side effects of anticholinergic agents include palpitations, nervousness, nausea, dry mouth, and cognitive dysfunction. In particular, trihexyphenidyl can impair cognitive function when administered at the standard clinical doses ${ }^{13)}$ as it crosses the blood-brain barrier. We examine the antispasmodic drug scopolamine butylbromide (SBB) as a treatment for CLZ-induced hypersalivation in a series of 5 patients. SBB is a synthetic quaternary ammonium compound with anticholinergic properties, which is chemically related to scopolamine, a synthetic tertiary compound. SBB exhibits increased lipid solubility compared with scopolamine; however, unlike synthetic tertiary compounds synthetic

(c) This is an Open-Access article distributed under the terms of the Creative Commons Attribution Non-Commercial License (http://creativecommons.org/licenses/by-nc/3.0) which permits unrestricted non-commercial use, distribution, and reproduction in any medium, provided the original work is properly cited. 
quaternary compounds such as SBB cannot penetrate the blood-brain barrier, limiting their central nervous system side effects. This study was designed to evaluate the efficacy and safety of using SBB to treat CLZ-induced hypersalivation.

\section{CASE}

Here a case series of five schizophrenia patients is presented who received outpatient or inpatient treatment at Okehazama Hospital from April 2012 to May 2013. Five patients were invited to participate in the trial if they were 20- to 65-years-old, met the Diagnostic and Statistical Manual of Mental Disorders 4th edition, text revision (DSM-IV-TR) criteria for schizophrenia or schizoaffective disorder, had been treated with CLZ for eight weeks, and were suffering from hypersalivation as show in Table 1. The study aims and methods were described to the subjects, and written informed consent was obtained from each of them. The study protocol was approved by the ethics committee at Okehazama Hospital. Five chronic schizophrenic patients who exhibited hypersalivation during CLZ treatment were coadministered SBB (30-60 mg/day) for 4 weeks.
At the baseline and after 4 weeks' treatment, hypersalivation was subjectively evaluated using a visual analog scale (VAS) and a face rating scale (FRS) and was objectively evaluated using the Drooling Severity Scale (DSS; 1: never drools, 2: mild drooling, 3: moderate drooling, 4: severe drooling, 5: profuse drooling) and Drooling Frequency Scale (DFS; 1: never, 2: occasionally, 3: frequently, 4: constantly). In addition, the Udvalg for Kliniske Undersogelser Side Effects Rating Scale (UKU) was used to identify side effects at the same time points. At the baseline and after 4 weeks' treatment, we used the Positive and Negative Syndrome Scale (PANSS) to assess psychological symptoms, the Wisconsin Card Sorting Test (WCST) to evaluate cognitive function, the Drug Attitude Inventory-10 Questionnaire (DAI-10) to examine medication adherence, and abdominal computed tomography (CT) scans to detect constipation.

The data of patients evaluation at baseline and weeks 4 demographic are presented in Table 2. Regarding the subjective evaluations of the patients' symptoms, none of the

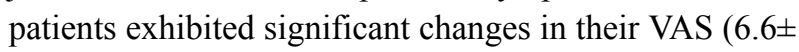
0.7 at the baseline vs. $5.7 \pm 1.1$ at week $4, p=0.277$ ) or FRS scores $(3.4 \pm 0.5$ at the baseline vs. $3.2 \pm 0.6$ at week 4 , $p=0.407$ ) after 4 weeks' treatment. Conversely, all 5 pa-

Table 1. Patients' background data

\begin{tabular}{|c|c|c|c|c|c|}
\hline No. & Sex & Age $(y r)$ & Disease duration (yr) & CLZ dosage (mg) & CLZ duration (mon) \\
\hline 1 & Female & 57 & 30 & 500 & 20 \\
\hline 2 & Female & 45 & 13 & 500 & 8 \\
\hline 3 & Female & 52 & 30 & 350 & 8 \\
\hline 4 & Female & 49 & 25 & 600 & 25 \\
\hline 5 & Male & 65 & 33 & 525 & 24 \\
\hline Total & & $53.6 \pm 7.7$ & $26.2 \pm 7.9$ & $495.0 \pm 90.8$ & $17.0 \pm 8.4$ \\
\hline
\end{tabular}

Values are presented as data only or mean \pm standard deviation.

CLZ, clozapine.

Table 2. Data for evaluated parameters

\begin{tabular}{|c|c|c|c|c|c|c|c|c|c|c|c|c|}
\hline \multirow{2}{*}{ No } & \multicolumn{2}{|c|}{ VAS } & \multicolumn{2}{|c|}{ FRS } & \multicolumn{2}{|c|}{ DSS } & \multicolumn{2}{|c|}{ DFS } & \multicolumn{2}{|c|}{ PANSS } & \multicolumn{2}{|c|}{ DAI-10 } \\
\hline & 0 Week & 4 Weeks & 0 Week & 4 Weeks & 0 Week & 4 Weeks & 0 Week & 4 Weeks & O Week & 4 Weeks & O Week & 4 Weeks \\
\hline 1 & 7.9 & 5.6 & 3 & 3 & 4 & 3 & 4 & 2 & 116 & 114 & -2 & 4 \\
\hline 2 & 6.7 & 10 & 3 & 5 & 4 & 3 & 4 & 3 & 88 & 72 & -2 & 8 \\
\hline 3 & 5 & 4.8 & 5 & 2 & 4 & 2 & 4 & 2 & 79 & 70 & 6 & 10 \\
\hline 4 & 8.1 & 3.5 & 4 & 4 & 4 & 2 & 4 & 2 & 93 & 85 & 4 & 2 \\
\hline 5 & 5.2 & 4.8 & 2 & 2 & 4 & 3 & 3 & 3 & 82 & 76 & 0 & 8 \\
\hline Total & $6.6 \pm 0.7$ & $5.7 \pm 1.1$ & $3.4 \pm 0.5$ & $3.2 \pm 0.6$ & $4.0 \pm 0.0$ & $2.6 \pm 0.2$ & $3.8 \pm 0.2$ & $2.4 \pm 0.2$ & $91.6 \pm 6.6$ & $83.4 \pm 8.1$ & $1.2 \pm 1.6$ & $6.4 \pm 1.5$ \\
\hline$p$-value & \multicolumn{2}{|c|}{0.277} & \multicolumn{2}{|c|}{0.407} & \multicolumn{2}{|c|}{0.002} & \multicolumn{2}{|c|}{0.012} & \multicolumn{2}{|c|}{0.011} & \multicolumn{2}{|c|}{0.968} \\
\hline
\end{tabular}

Values are presented as data only or mean \pm standard deviation.

VAS, visual analog scale; FRS, face rating scale; DSS, Drooling Severity Scale; DFS, Drooling Frequency Scale; PANSS, Positive and Negative Syndrome Scale; DAl-10, Drug Attitude Inventory-10 Questionnaire.

The data were analyzed using paired t-test regarding changes from baseline measurement. A p-value of $<0.05$ was defined as statistically significant. 
tients displayed significant changes in their DSS (4.0 \pm 0.0 at the baseline vs. $2.6 \pm 0.2$ at week $4, p=0.002$ ) and DFS scores $(3.8 \pm 0.2$ at the baseline vs. $2.4 \pm 0.2$ at week 4 , $p=0.012$ ) after 4 weeks' treatment.

As for psychopathological symptoms, all 5 patients exhibited significant changes in their total PANSS scores after 4 weeks' treatment $(91.6 \pm 6.6$ at the baseline vs. 83.4 \pm 8.1 at week $4, p=0.011$ ). According to the UKU, no SBB-related side effects developed after the baseline. However, a CT scan performed after 4 weeks' treatment revealed air pockets in patient 4's abdomen. Three patients were able to use the WCST to assess their cognitive dysfunction; however, no worsening of cognitive dysfunction was detected in any case.

\section{DISCUSSION}

CLZ has anticholinergic properties, and dry mouth is commonly experienced as a side effect of anticholinergic treatment. Thus, hypersalivation is a somewhat unexpected side effect of CLZ treatment. In animals, olanzapine (OLZ) has been shown to have a similar pharmacological profile to CLZ. ${ }^{14)}$ However, whilst OLZ has often been reported to cause dry mouth, it rarely induces hypersalivation. Tollefson et al. ${ }^{15)}$ reported that a significantly greater proportion of CLZ-treated patients than OLZ-treated patients experienced hypersalivation. Weiner et al. ${ }^{16)}$ also compared CLZ and OLZ, both of which are antagonists of M1-M5 receptors, and found that CLZ has stronger antagonistic effects on M1, M3, and M5 receptors than OLZ. It should be noted that CLZ also has agonistic effects on M1, M2, and M4 receptors. Also, profiling has revealed that $\mathrm{N}$-desmethylclozapine (NDMC), the principal metabolite of CLZ, but not CLZ itself, is a potent and effective muscarinic receptor antagonist and agonist. NDMC has agonistic effects on M1-M5 receptors and antagonistic effects on the M3 receptor. CLZ-induced hypersalivation is hypothesized to be predominately mediated by antagonistic and agonistic effects on muscarinic receptors. SBB is a derivative of scopolamine. However, SBB cannot cross the blood-brain barrier. Also, SBB is a non-subtype-selective muscarinic receptor antagonist. ${ }^{17)}$ Therefore, we considered that SBB might be useful for treating CLZ-induced hypersalivation. This study demonstrates the efficacy of SBB (30-60 mg/day) against CLZinduced hypersalivation. Whilst subjective assessments based on a VAS and a FRS did not detect any significant differences in the extent of hypersalivation between the baseline and week 4, objective assessments based on the
DSS and DFS did detect such differences. This possibility is that the patients had excessive expectations regarding the effects of SBB. The patients' DSS and DFS scores differed significantly between the baseline and week 4; however, none of the patients exhibited DSS or DFS scores of 0 at week 4. Patients find hypersalivation quite distressing; therefore, it might be associated with a considerable reduction in QOL. Hence, the fact that SBB treatment did not result in the complete remission of the patients' hypersalivation might explain the results obtained with the VAS and FRS. Many previous studies and our study have found that muscarinic receptor antagonists had positive effects on hypersalivation, it should be noted that none of these agents induced complete remission.

In the present study, SBB treatment was associated with a significant improvement in the patients' psychopathology, as measured on the PANSS. Improvements were also observed in items associated with the PANSS general score. Thus, hypersalivation might have exacerbated the patients' psychopathology as well as decreased their QOL. The relationships between particular side effects and psychopathology need to be verified in future studies. Thus, medical staff who treat hypersalivating patients with SBB should always inquire about the extent of their hypersalivation before and after treatment. We chose SBB for this study because it does not cross the blood-brain barrier; thus, it causes fewer anticholinergic side effects such as mouth dryness, constipation or changes in blood pressure. None of these side effects were detected by the UKU in the present study. In one patient, a CT scan performed after 4 weeks' treatment detected abdominal air pockets. This was considered to be a side effect of the interaction between CLZ and SBB and their anticholinergic effects. The patient who developed abdominal air pockets was taking $60 \mathrm{mg} /$ day SBB. Therefore, we might expect the frequency of abdominal air pockets to rise as the dose of SBB increases from 30 to $60 \mathrm{mg}$. We therefore suggest that SBB can be used safely providing adequate clinical observation for possible side effects is employed.

In conclusion, the efficacy and safety of using SBB to treat CLZ-induced hypersalivation were confirmed in this trial. Further data are needed to confirm the findings of this trial, and well-designed randomized controlled trials would be ideal for this purpose.

\section{REFERENCES}

1. Davydov L, Botts SR. Clozapine-induced hypersalivation. Ann Pharmacother 2000;34:662-665.

2. Zeng XP, Le F, Richelson E. Muscarinic $m 4$ receptor activation by some atypical antipsychotic drugs. Eur $J$ 
Pharmacol 1997;321:349-354.

3. Kishi T, Fujita K, Furukawa O, Suzuki T, Moriwaki M, Nitta $\mathrm{M}$, et al. Efficacy and tolerability of clozapine in Japanese patients with treatment-resistant schizophrenia: results from a 12-week, flexible dose study using raters masked to antipsychotic choice. Asian J Psychiatr 2013; 6:200-207.

4. Nielsen J, Dahm M, Lublin H, Taylor D. Psychiatrists' attitude towards and knowledge of clozapine treatment. $J$ Psychopharmacol 2010;24:965-971.

5. Li Z, Huang M, Ichikawa J, Dai J, Meltzer HY. N-desmethylclozapine, a major metabolite of clozapine, increases cortical acetylcholine and dopamine release in vivo via stimulation of M1 muscarinic receptors. Neuropsychopharmacology 2005;30:1986-1995.

6. Zorn SH, Jones SB, Ward KM, Liston DR. Clozapine is a potent and selective muscarinic M4 receptor agonist. Eur J Pharmacol 1994;269:R1-2.

7. McKane JP, Hall C, Akram G. Hyoscine patches in clozapine-induced hypersalivation. Psychiatr Bull 2001; 25:277.

8. Calderon J, Rubin E, Sobota WL. Potential use of ipatropium bromide for the treatment of clozapine-induced hypersalivation: a preliminary report. Int Clin Psychopharmacol 2000; 15:49-52.

9. Schneider B, Weigmann H, Hiemke C, Weber B, Fritze J. Reduction of clozapine-induced hypersalivation by pirenzepine is safe. Pharmacopsychiatry 2004;37:43-45.

10. Spivak B, Adlersberg S, Rosen L, Gonen N, Mester R, Weizman A. Trihexyphenidyl treatment of clozapineinduced hypersalivation. Int Clin Psychopharmacol 1997;
12:213-215.

11. Praharaj SK, Sarkhel S, Khanande RV, Sinha VK. Complete resolution of clozapine-induced sialorrhea with low dose trihexyphenidyl. Psychopharmacol Bull 2010;43:73-75.

12. Leung JG, Puri NV, Jacobson MJ. Immediate-release oxybutynin for the treatment of clozapine-induced sialorrhea. Ann Pharmacother 2011;45:e45.

13. Ancelin ML, Artero S, Portet F, Dupuy AM, Touchon J, Ritchie K. Non-degenerative mild cognitive impairment in elderly people and use of anticholinergic drugs: longitudinal cohort study. BMJ 2006;332:455-459.

14. Bymaster FP, Calligaro DO, Falcone JF, Marsh RD, Moore $\mathrm{NA}$, Tye NC, et al. Radioreceptor binding profile of the atypical antipsychotic olanzapine. Neuropsychopharmacology 1996; 14:87-96.

15. Tollefson GD, Birkett MA, Kiesler GM, Wood AJ; Lilly Resistant Schizophrenia Study Group. Double-blind comparison of olanzapine versus clozapine in schizophrenic patients clinically eligible for treatment with clozapine. Biol Psychiatry 2001;49:52-63.

16. Weiner DM, Meltzer HY, Veinbergs I, Donohue EM, Spalding TA, Smith TT, et al. The role of M1 muscarinic receptor agonism of $\mathrm{N}$-desmethylclozapine in the unique clinical effects of clozapine. Psychopharmacology (Berl) 2004; 177:207-216.

17. Raufman JP, Shant J, Xie G, Cheng K, Gao XM, Shiu B, et al. Muscarinic receptor subtype-3 gene ablation and scopolamine butylbromide treatment attenuate small intestinal neoplasia in Apcmin/+ mice. Carcinogenesis 2011;32: 1396-1402. 